\title{
Morphology of toxic metal distributions in biological soft tissue of deep-sea hydrothermal extremophile polychaete Alvinella pompejana
}

\section{LAUREL R YOHE AND DREW D SYVERSON}

Yale University

Presenting Author: loloyohe@gmail.com

Hydrothermal vent systems provide some of the most extreme environments on the planet, including extreme ranges in temperature, pressure, anoxic conditions, and exposure to toxic metals. However, the role of eukaryotic fauna and their interactions with extremophile prokaryotes in the biogeochemical cycling of toxic metals released from the vent systems is not well understood. Quantifying the extent to which metal species get "trapped" on the seafloor after immediately being released from the vent fluids is crucial to understanding metal distributions on a global scale and potential impact on the geological time scale. Alvinella pompejana, or the Pompeii worm, is a deep-sea polychaetae worm that is arguably among the most extreme-adapted marine invertebrates. In addition to high heat and anoxia, Alvinella also copes with $\mathrm{H}_{2} \mathrm{~S}$ concentrations beyond what would kill a human, as well as a number of other toxic metals (e.g. $\mathrm{Hg}, \mathrm{Pb}, \mathrm{As}, \mathrm{Cd}$ ). Several lines of defense likely contribute to the detoxification of the hydrothermal environment for the worm to survive, including secreted tubes to selectively diffuse compounds or epibiotic metal-resistant bacteria that may act as a "metal sunscreen". However, the tissues of both the body and tentacles have high concentrations of heavy metals that differ significantly in reducible or oxidized state, depending on the region of the worm. This suggests that the spatial distribution of minerals present within the tissue of the Alvinella are different regions of the animal metabolize and detoxify different metals and compounds. We applied pioneering imaging techniques including diffusible iodine-based contrast-enhanced $\mu \mathrm{CT}$-scanning (diceCT) and laser-induced breakdown spectroscopy (LIBS) to understand the morphological distribution of different toxic metals in the soft tissues of Alvinella. Visualizing metal distributions in three dimensions offers a pioneering approach to quantifying the geobiological role of deep-sea organisms in metal cycling. 